\title{
Routing Approaches for Cognitive Radio Ad-hoc Networks and Challenges
}

\author{
Harshal Solao \\ Dept. of Computer Engineering \\ MIT Academy of Engineering \\ University of Pune, India
}

\author{
R. M. Goudar \\ Dept. of Computer Engineering \\ MIT Academy of Engineering \\ University of Pune, India
}

\author{
Sunita Barve \\ Dept. of Computer Engineering \\ MIT Academy of Engineering \\ University of Pune, India
}

\begin{abstract}
Cognitive radio networks (CRNs) are composed of cognitive devices capable of changing their configurations on Real time, based on the spectrum environment. This capability provides chance to opportunistically reuse the portion of the spectrum as required resigned by the licensed users (PU's). From another point of view, the tractability in the spectrum access level attaches to an increased complexity for designing of communication protocols at each layer. This brings focuses on the problem of designing efficient routing results for multihop CRNs that become a central issue in cognitive networking paradigm. We furnish a broad overview of the research in the area of routing for CRNs, distinguishing two main categories: full spectrum knowledge base, and local spectrum knowledge base procedures and protocols. For each category we depict on proposed design methodologies and routing metrics. Finally, potential future research directions are also suggested.
\end{abstract}

\section{General Terms}

Algorithms, Methods, Techniques, Approaches

\section{Keywords}

Cognitive radio Networks (CRN), Classification in routing, centralized and Decentralized routing, Multi-hop Networks, Global and Local knowledge base routing.

\section{INTRODUCTION}

Fixed spectrum assignment policies in current wireless networks are regulated by governmental agencies. Licenses are provided for the use of various, often comparatively small, frequency bands on a long term basis over large geographical regions. Today enormous use of wireless applications and Services has caused an exponential increase in requests to regulatory agency for spectrum allocation. The uses of wireless technologies working in unlicensed bands, like ISM band, become overcrowded by various applications developed in different fields. From another point of view, the use of licensed spectrum is totally depends on specific wireless technology, their market requirement and their customer services to which band have been assigned. As stated in recent studies by the Federal Communications Commission (FCC) the spectrum band which are allocated through fixed assignment policies are used for particular geographical area or over fixed periods of time, due to which the average utilization of these band varies between $15 \%$ and $85 \%[1,2]$.

To overcome this situation, the impression of dynamic spectrum access (DSA) has been suggested. With these, licensed spectrum bands may use by unlicensed users opportunistically without interfering license users. In technical point of view, this is possible only because of recent advancement in the area of software-defined radios (SDRs) that allow cognitive devises to reconfigure to operate on a wide spectrum band and tuned to required frequency band in the range with minimum delay [3,4]. Resulting so-called Cognitive Radio (CR) devices have the capability to change their transmitting parameters i.e. operating spectrum, modulation, transmission power, and communication technology based on interaction with spectral environment. Cognitive radio devises can sense a large spectrum band dynamically to identify idle spectrum blocks for transmission and accessing the unused spectrum called Spectrum Opportunities (SOP) [6] intelligently.

Cognitive Radio Networks (CRNs) are created by using devices with cognitive capabilities that provide solution to address the fixed spectrum accessibility and the inefficiency in the spectrum usage [6]. In general CRNs distinguishes the sharing a common spectrum within two types of users with different rules: Primary User (PUs) or Licensed User have highest priority to use the spectrum within licensed band and Secondary User (SUs) or Unlicensed User must access spectrum opportunistically. PUs use conventional wireless communication systems with static spectrum allocation. SUs are equipped with CRs and exploit Spectrum Opportunities (SOPs) to maintain their activities without interfering with PU transmissions.

An issue on Physical layer and /or Medium Access Control in CRNs has concentrated on single-hop scenarios by considering spectrum management techniques [7, 8]. Recently the research community has started realizing the importance of multi-hop CRNs that can widely use in various fields.

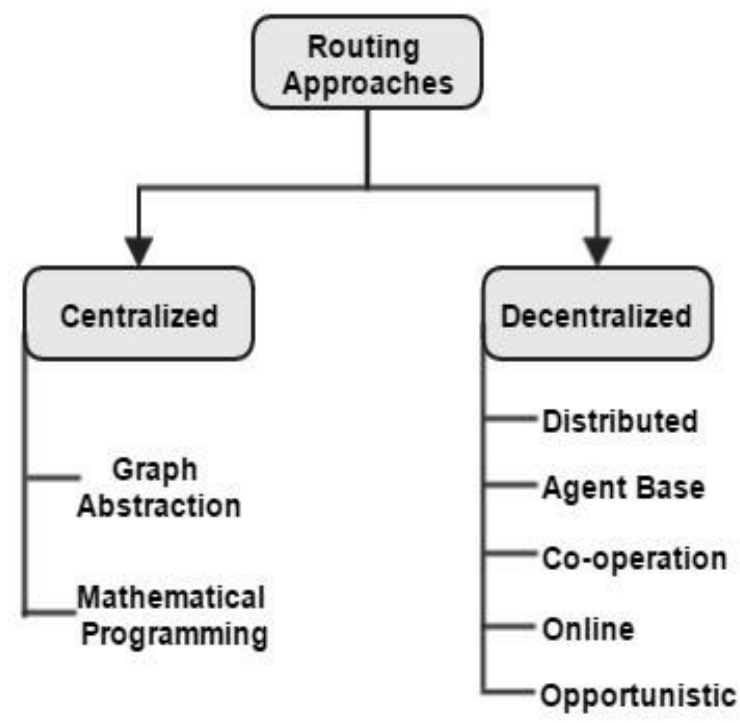

Figure 1: Cognitive routing approach 
The remaining paper is focus on the centralized and decentralized routing approach in CRNs as shown in Figure 1; it also discusses challenges and future direction. These approaches are also known as Global and Local spectrum knowledge based Routing schemes.

\section{CENTRALIZED ROUTING APPROACH}

In Centralized Routing approach, centrally-maintained spectrum data bases are used to stores the channel availabilities over a time [9]. Before data sending and receiving, cognitive devices will check the central databases for channel availability. In this centralized routing approach spectrum sensing and sharing are completely separated task. Generally, centralized spectrum access requires the being of a central node that executes most of the activities and takes decisions to assigning the channels to the cognitive devices. This central device may be a separate network entity called Spectrum Server, Broker, or a central base station that gathers spectrum and radio info from all SUs as required.

A centralized spectrum access has various advantages on account of the global spectrum info that the spectrum broker has, that become more well to increase the overall throughput and to decrease interference between SUs and in the network functioning.

The routing approaches that build on this centralized supposition leverage theoretical tools like Graph Abstraction, Mathematical Programming etc., [10] because they provide upper bound and basic standard for the routing performance. Some of the routing approaches belong to centralized entity are as follows:

\subsection{Routing based on Graph Abstraction}

\subsubsection{Based on DAG (Directed acyclic graph)}

Graph based routing become a topological due to its dependencies among the node like Tree based, Directed acyclic graph based. However, the inherent limitation of the tree-based approach is that it utilizes only directed edges to route to a destination, where denotes the number of nodes in the network. Multipath routing is a best routing scheme to adapt these requirements by using multiple pairs of routes from a source to a destination.

The main aim is hence to employ the extra links available in the network to ameliorate robustness. For this we have to construct independent directed acyclic graphs rooted at each node [13]. Techniques used for multipath routing are frequently based on utilizing multiple spanning trees or directed acyclic graphs (DAGs).

When numbers of routing tables are used, packet needs to carry in its header which routing table to be used for this. When the adjacent next edge is not available, this packet must be dropped. This is because of the possible looping of packets when transmitted from one routing table to another. In the case of DAGs, calculated by contributing edges to the shortest-path tree, it can't guarantee that a single-link failure will not disconnect nodes from the destination.

To accomplish resilient multipath routing, author [13] bring in the concept of independent directed acyclic graphs (IDAGs). In which the Link-independent (node-independent) DAGs meet the property that any path between a source and the root on one DAG is link-disjoint (node-disjoint) on other path between the source and the root on the other DAG. The linkindependent DAGs meet the following property

$$
\forall_{\boldsymbol{s}} \in \boldsymbol{N}, \boldsymbol{s} \neq \boldsymbol{d} \boldsymbol{i} \rightarrow \boldsymbol{j} \in \boldsymbol{P}_{\boldsymbol{s d}}=>i \rightarrow j, j \rightarrow i \nexists \boldsymbol{P}_{\boldsymbol{s d}}^{\prime}
$$

The node-independent DAGs must fulfill the below property:

$$
\forall_{s} \in N, s \neq d i \neq s, d \quad i \in P_{s d}=>i \nexists P_{s d}^{\prime}
$$

Where, $i \rightarrow j, j \rightarrow i$ is directed links, DAG is rooted at d, $\boldsymbol{P}_{\boldsymbol{s} \boldsymbol{d}}$ and $\boldsymbol{P}_{\boldsymbol{s} \boldsymbol{d}}$ denotes any two paths from $\mathbf{s}$ to $\mathbf{d}$ one from each DAG.

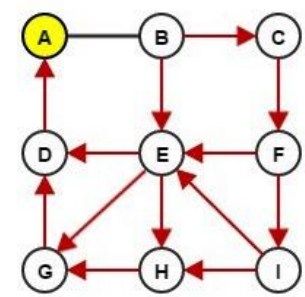

(a)

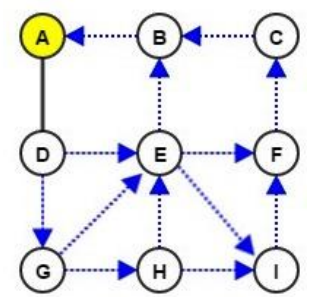

(b)
Figure 2: (a) and (b) shows two independent directed acyclic graphs rooted at node $A$.

In Figure 2(a) and 2(b) Consider the path from node $\mathrm{F}$ to $\mathrm{A}$ on blue DAG. If link $\mathrm{C}$ to $\mathrm{B}$ fails then packet is rerouted on red DAG on F-I-H-G-D-A even though the link I-H fails packet may be sent on link I-E to reach at destination. Some of the graph base routing like layered graph, colored graph are describe in [12].

\subsubsection{Based on Clustering}

Opportunistic way to utilize spectrum bands comes with the some important challenges including routing. Several approaches have been introduced one of the approach is dividing the network into segments of small size this also known as clustering.

It can be two types, cluster with or without cluster head (CHs) as shown in Figure 3, CHs may do for many purpose within cluster like allocation of resources to cluster nodes and coordination between them [14]. CHs controlled cluster can be organized as either one hop or k hop (multi hop) clusters.

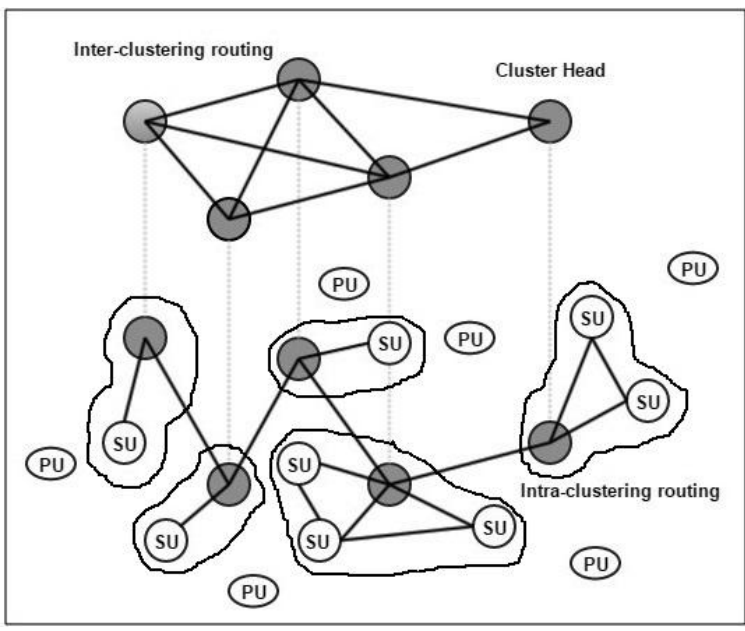

Figure 3: Cluster Connectivity

It is presumed that there is no link (i.e. no cooperation) between primary and secondary networks. A novel algorithm, united nodes (UNITED), is proposed in [15] for maximizing the network throughput and minimizing the end-to-end delay.

The UNITED operates at every node. Initially, nodes organize themselves into several clusters by the clustering algorithm 
that is based on central location. Following completion of cluster establishment, routing is behaved allowing to the spectrum usage and interference metrics. Clusters adapt themselves centrally with respect to spectrum availability.

\subsection{Routing based on Mathematical Abstraction}

In [16] authors suggest joint time division multiple access (TDMA) MAC scheduling and channel assignment. In which first they formulate a centralized algorithm that employs the concept of "independent link sets" for scheduling slots and routing through channel allocation.

The proposed centralized algorithm base on linear programming for one hops communication. The centralize MAC Time/Channel Assignment are formulated for network perspective. This approach is beneficial to infrastructure (topological) based domain.

\section{LIMITATIONS OF CENTRALIZED ROUTING}

Even though there are different methods and techniques that work considerably and improve the centralized routing approach as go through above, some of the limitations are discussed below:

- Central entity must have perfect knowledge of network topology.

- Communication is done by accessing central databases for spectrum statistics.

- Each node is not able to take its own routing decisions.

- Solution based on theoretical tools like Graph Abstraction, mathematical programming provides upper bound and benchmarks for routing performance.

- $\quad$ Provide static cognitive multi hop network.

- It separates the Sharing and Sensing functionality.

These limitations will motivate us to use Decentralized (Local spectrum information base) routing approach discussed underneath including their challenges.

\section{DECENTRALIZED ROUTING APPROACH}

The limitations of centralized routing approach are overcome by achieving challenges in decentralized routing approach.

- Distributed

Every activity should be standalone like Channel availability calculation, information sharing, selecting relay node etc.

- $\quad$ Agent base

The meaning of Agent base is that the each node/device should be able to take its own decision and Formulation.

- Co-operation

The each node must share their own information to other neighbors. That will be useful for effective routing.

- Online

It should be online that the each node takes its decision as per the current environment availability.

\section{- Opportunistic}

Whenever there is vacant space available it should take this opportunity for communication.

Keeping eye on these challenges we are going discuss different decentralized routing approach.

\subsection{Distributed Routing Approach}

This gives information on routing solution where the spectrum occupancy information is performed in a distributed way like classical ad-hoc networks.

As an example in [17] author introduce new routing scheme "Gymkhana" for CRAHNs which is aware of degree of connectivity of possible paths towards the destination. It selects the path having less number of PUs that gives high stability and robustness to the route. Laplacian matrixes are used to compute the connectivity of different networks paths.

For path connectivity in CRAHN, Gymkhana uses:

- A distributed AODV style protocol to collect some key parameter.

- Use basic mathematical structure and representing graph.

Gymkhana Algorithm:

Processing of the contents of received RREQ (route request) at destination is done in two steps:

1. Formation of virtual graph $\mathrm{V}_{\mathrm{k}}$ for each of the $\mathrm{k}$ path

2. Calculation of the eigenvalues of the laplacian of the virtual graph.

The number of nodes of $\mathbf{V}_{\mathbf{k}}$ is equal to

$$
\mathbf{M}_{\mathrm{k}=}\left(\mathbf{H}_{\mathrm{k}+1}\right) * \mathbf{N}_{\mathbf{p}}
$$

Where, $\mathrm{k}$ is number of possible path, $\mathbf{H}_{\mathbf{k}}$ is number of hops in the k-th path and $\mathbf{N}_{\mathbf{p}}$ denote virtual nodes. Therefore among the $\mathbf{k}$ possible paths the destination node selects the one with highest value of Utility function which depends on Eigen values, when there are not PUs influencing nodes of the path k.

Routing protocol can be designed to distribute routing as per requirements like Class I routes that gives better performance, other Class II routes which achieves a higher amount of security for PUs.

In class I route end-to-end latency in the cognitive radio network is main consideration where maximum propagation distance and most farseeing allowed duration for transmission is selected by spectrum [18]. The class II route is product of minimum fractional area of overlap between cognitive and PUs vicinity.

The active route maintenance is done by the secondary user by supervising its own position with respect to primary user position. If the movement of PUs is towards SUs then it discover new path as a precaution for route failure.

\subsection{Agent base Co-operative Routing Approach}

An artificially designed ANT colony (see Figure 4) can be used for detecting, noticing and finding out routing schemes by guided ants communicating in an indirect path [19]. 
In this routing each node maintains its own local heuristic information and history. Spectrum between any node $\mathbf{n}$ with its neighbor $\mathbf{x}$ can be qualified as local heuristic info $\mathbf{~}_{\mathbf{x}}^{\mathbf{n}}$. Info of the history path quality between $\mathbf{n}$ and $\mathbf{y}$ is presented using $\boldsymbol{\mu}_{\boldsymbol{y}}^{\boldsymbol{n}}$ and $\boldsymbol{\tau}_{\boldsymbol{x} \boldsymbol{y}}^{\boldsymbol{n}}$ shows the preferences of taking node $\mathbf{x}$ when destination is $\mathbf{y}$.

The each node in the colony share information about spectrum availability to its neighbor over common control channel. If the neighbor info is not available the forward ant sent it in broadcast way otherwise it send in unicast way.

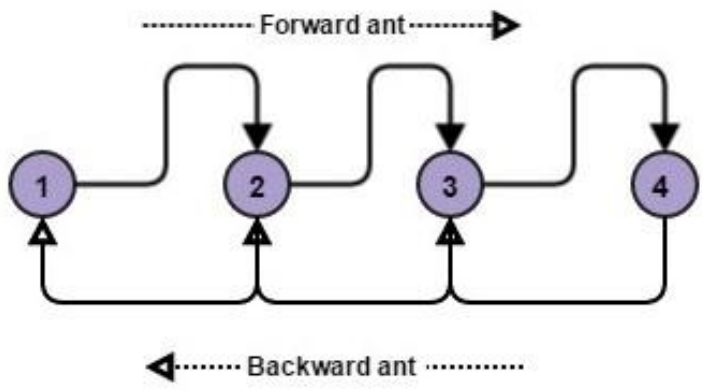

Figure 4: Information exchange using Ants

The probability of node $\mathbf{n}$ chooses $\mathbf{x}$ as next relay node towards destination $\mathbf{d}$ is:

$$
\operatorname{Prob}_{x d}^{n}=\frac{\left(\tau_{n d}^{n}\right)^{\alpha} \cdot\left(l_{x}^{n}\right)^{\beta}}{\sum_{i \in N_{e i}(n)}\left(\tau_{n d}^{n}\right)^{\alpha} \cdot\left(l_{x}^{n}\right)^{\beta}}
$$

Where $\boldsymbol{\alpha}$ and $\boldsymbol{\beta}$ represent the adjustable weights of pheromones concentration and local heuristics.

After reaching forward ant to the destination, backward ant keeps tracking record of local heuristic info and modify routing table along their paths.

Other example, the usefulness of game theory and optimal methods are provided in [20] also become effective solution of competitor pricing and marketing model problem for spectrum sharing and allotment.

The proposed Hierarchical spectrum sharing network (HSSN) consist of infrastructure based and ad-hoc cells is mixed with economical paradigm to form spectrum framework for utility maximization by prizing the spectrum that used for communication with other destinations between a number of SUs and base station within a cell. Every base station determines the prices of the spectrum that each SU uses for transmission as an administrator. Each SU builds an allotment of its spectrum itself and granting to its utility maximization determines the relay node carried to.

\subsection{Online Routing Approach}

The online work consideration means that the every CR node have information about its environment like number of channels, there quality to allocate resources for data communication. IP spectrum aware routing protocol takes this all required information from sensing output of the lower two layers [21].

The routing is done by choosing best channel for forwarding data packet from current node to next relay node with QoS. Channel selection is based on common channel sensed by both nodes.
The given IPSAG (IP Spectrum Aware Geographic) routing works on two modes:

\section{IPSAG algorithm based routing}

2. Inside preexisting system (WIFI, WIMAX etc.,) according to existing protocol it known as a tunneling.

It uses local information of available channels and node position, according to that it takes decision for online hop-byhop geographic routing.

\subsection{Opportunistic Routing Approach}

As we know that the cognitive routing is coupled with spectrum sensing and sharing in multi hop, multi-channel cognitive radio network. The spectrum band is dynamic in nature in which the unused spectrum can be utilized opportunistically. The opportunistic use of such spectrum band for routing is become opportunistic routing.

The author in [22] proposed the Opportunistic cognitive routing (OCR) protocol which is selects the forwarding links on the basis of local statistics of spectrum access opportunities. The new routing metric is also proposed for catching unequaled properties of $\mathrm{CRN}$, denoted as a cognitive transport throughput (CTT).

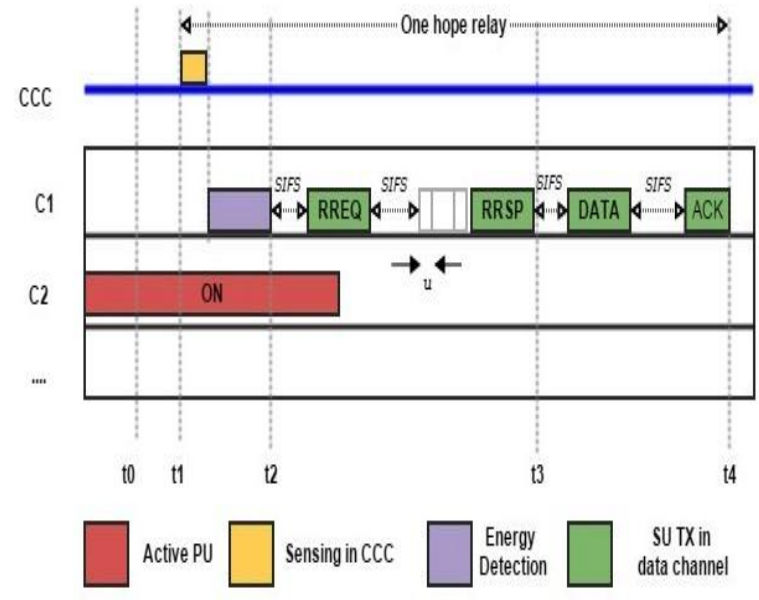

Figure 5: One hope relay in OCR time line

They consider set of channel $\boldsymbol{C}$ share among all PUs and SUs over common control channel (CCC). The one hop relay OCR time line is shown in Figure 5 the channel usage statistics is obtained by periodic sensing operation of ON (PU active) and OFF (PU inactive) signals. It also proposed heuristic algorithm to reduce searching complication of the optimum selection of channel and relay.

\section{DISCUSSION AND FUTURE SCOPE}

In this paper, we present an overview of centralized (full spectrum knowledge) and decentralized (local spectrum knowledge base) routing approaches in CRNs. We discussed existing work for routing in multi-hop CRNs. The limitation of centralized routing and challenges of decentralized routing are also defined by considering the stochastic environment in to account

On the basis of their working environment we divide it into two main categories i.e. centralized (Global Spectrum Information Based) Routing schemes and decentralized (Local Spectrum Information Based) Routing schemes. 
Cognitive radio network are well versed users that are easily learn to achieve optimal performance. In context of routing scheme we believe that there is future work necessitated to designing precise routing approach. Every scheme must be consider both global and local metric to route formation. Below, we summaries some open research issues for routing in CRNs:

\subsection{Routing Path Reconfiguration}

As we discussed earlier the environment is highly dynamic because of PUs. After route formations the route failure becomes more common due to dynamicity. In such case reconfiguration of route is necessary but it become overhead for routing protocol to reconfigure route.

If the routing algorithm is aware about spectrum statistics that will help to reduce the re-routing overhead that means it must know the selected node become more stable and fruitful this can be accomplishable by referring history information about selected node and real time learning.

Our view on this topic is that the use of machine learning technique where exploitation and exploration become fundamental functionality is more useful to solve this type of problem.

\subsection{Best Relay Node Selection}

Now a day many routing solution use information sharing methods for getting the local spectrum statistics for selecting common control channel between source to destination which badly affects the overall network performance.

Instead of selecting common control channel between source to destination if they (each node) selects best available channel between current node and next relay node as a control channel and other as a data channel it will be more useful and effective for network performance as well as selecting best stable relay node.

\subsection{Calculating Channel Availability}

In decentralized environment channel availability is calculated by sensing the spectrum environment to find out unused spectrum that can be used in an opportunistic manner.

In this highly mobile and dynamic spectrum instated of calculating channel availability online manner in real time if we calculate it before time that will become more effective.

This can be achievable with the help of artificial intelligence and prediction models using prediction models which will calculate the channel availability prior time.

\section{CONCLUSION}

Routing in CRN is significant area of research as it provides various aspects to discover innovative ideas. As its works on spectrum management and spectrum aware routing it requires routing protocols which able to learn to solve different problem dynamically.

In this routing survey, we have discussed existing work for routing in multi-hop CRNs. The limitation of centralized routing and unequaled challenges of decentralized routing are also defined by considering the stochastic environment. A categorization of routing approaches in context of their knowledge base (i.e. Global and Local) and their future in our view.

\section{REFERENCES}

[1] F.C. Commission, Spectrum policy task force, Technical report, November 2002.
[2] I.F. Akyildiz, Y. Altunbasak, F. Fekri, R. Sivakumar, AdaptNet: adaptive protocol suite for next generation wireless internet, IEEE Communications Magazine 42 (3)(2004) 128-138

[3] E. Buracchini, The software radio concept, IEEE Communication Magazine 38 (9) (2000) 138-143.

[4] J. Mitola, Cognitive radio, An Integrated Agent Architecture for Software Defined Radio, $\mathrm{PhD}$ Dissertation Thesis, KTH, Sweden.

[5] K. Chowdhury, I. Akyildiz, Cognitive wireless mesh networks with dynamic spectrum access, IEEE Journal on Selected Areas in Communications 26 (1) (2008) 168-181, doi:10.1109/JSAC.2008.080115.

[6] I.F. Akyildiz, W.-Y. Lee, M.C. Vuran, S. Mohanty, Next generation/dynamic spectrum access/cognitive radio wireless networks: a survey, Computer Networks 50 (13) (2006) 2127-2159. doi: 10.1016/j.comnet.2006.05.001 .

[7] C. Cormio, K.R. Chowdhury, A survey on MAC protocols for cognitive radio networks, Ad Hoc $\begin{array}{lllll}\text { Networks } & 7 & \text { (7) (2009) 1315-1329, }\end{array}$ doi:10.1016/j.adhoc.2009.01.002.J

[8] S. Haykin, J.H. Reed, G.Y. Li, M. Shafi, Scanning the issue, Proceedings of the IEEE 97 (5) (2009) 784-786, doi:10.1109/JPROC.2009.2015701.

[9] Cesana, Matteo, Francesca Cuomo, and Eylem Ekici. "Routing in cognitive radio networks: Challenges and solutions." Ad Hoc Networks 9.3 (2011): 228-248.

[10] I.F. Akyildiz, W.Y. Lee, M. Vuran, S. Mohanty, A survey on spectrum management in cognitive radio networks, IEEE Communication Magazine 46 (4) (2008) 40-48.

[11] FCC, FCC 08-260, Unlicensed Operation in the TV Broadcast Bands, November 2008.

[12] C. Xin, B. Xie, C.-C. Shen, A novel layered graph model for topology formation and routing in dynamic spectrum access networks, in: First IEEE International Symposium on New Frontiers in Dynamic Spectrum Access Networks, DySPAN 2005, pp. 308-317. doi:10.1109/DYSPAN.2005.1542647.

[13] Cho, Sangman, Theodore Elhourani, and Srinivasan Ramasubramanian. "Independent directed acyclic graphs for resilient multipath routing." IEEE/ACM Transactions on Networking (TON) 20.1 (2012): 153-162.

[14] Islam, AKM Muzahidul, et al. "An efficient routing protocol on a Dynamic Cluster-based Sensor Network." Cognitive Radio Oriented Wireless Networks and Communications (CROWNCOM), 2011 Sixth International ICST Conference on. IEEE, 2011.

[15] Talay, A. Cagatay, and D. Turgay Altilar. "United nodes: cluster-based routing protocol for mobile cognitive radio networks." IET communications 5.15 (2011): 2097-2105.

[16] Younis, O.; Shallcross, D.; Kant, L.; Young, K.; Graff, C.; Patel, M., "TDMA scheduling and channel assignment for cognitive tactical networks," MILITARY COMMUNICATIONS CONFERENCE, 2012 MILCOM 2012 , vol., no., pp.1,6, Oct. 29 2012-Nov. 1 2012

doi: 10.1109/MILCOM.2012.6415665 
[17] Abbagnale, Anna, and Francesca Cuomo. "Gymkhana: a connectivity-based routing scheme for cognitive radio ad hoc networks." INFOCOM IEEE Conference on Computer Communications Workshops, 2010. IEEE, 2010.

[18] Chowdhury, K.R.; Akyildiz, I.F.; , "CRP: A Routing Protocol for Cognitive Radio Ad Hoc Networks," Selected Areas in Communications, IEEE Journal on , vol.29, no.4, pp.794-804, April 2011, doi: 10.1109/JSAC.2011.110411

[19] Bowen Li; Dabai Li; Qi-hui Wu; Haiyuan Li; , "ASAR: Ant-based spectrum aware routing for cognitive radio networks," Wireless Communications \& Signal Processing, 2009. WCSP 2009. International Conference on , vol., no., pp.1-5, 13-15 Nov. 2009, doi: 10.1109/WCSP.2009.5371704
[20] Nan Li; Lei Gong; Shaoqian Li; , "Price-based spectrumallocation relay routing in cognitive radio networks," Information, Communications and Signal Processing, 2009. ICICS 2009. 7th International Conference on , vol., no., pp.1-5, 8-10 Dec. 2009, doi: 10.1109/ICICS.2009.5397471.

[21] Bădoi, C.-I.; Croitoru, V.; Prasad, R.; , "IPSAG: An IP Spectrum Aware Geographic Routing algorithm proposal for multi-hop Cognitive Radio networks," Communications (COMM), 2010 8th International Conference on , vol., no., pp.491-496, 10-12 June 2010 doi: 10.1109/ICCOMM.2010.5509020

[22] Liu, Yongkang, Lin X. Cai, and Xuemin Shen. "Spectrum-aware opportunistic routing in multi-hop cognitive radio networks." Selected Areas in Communications, IEEE Journal on 30.10 (2012): 19581968. 